\title{
Psychological, situational and application- related determinants of the intention to self-test: a factorial survey among students
}

\author{
Pinar Kuecuekbalaban *iD, Tim Rostalski, Silke Schmidt and Holger Muehlan
}

\begin{abstract}
Background: The Internet enables an unprecedented opportunity to access a broad range of self-tests (e.g. testing for HIV, cancer, hepatitis B/C), which can be conducted by lay consumers without the help of a health professional. However, there is only little knowledge about the determinants of the use of self-tests. Thus, the aims of this study were (1) to experimentally investigate the impact of situational and application-related characteristics on the intention to use a self-test (ST), compared to being tested by a health professional at home (HPH) or at a doctor's office (HPD), (2) to examine the applicability of social-cognitive health behaviour theories on selftesting, and (3) to explore the advantages of integrating technological affinity into social-cognitive health behaviour models to predict self-testing.

Methods: In a factorial survey, 1248 vignettes were rated by 208 students. The core concepts of socialcognitive health behaviour theories, technological affinity, and different situational and application-related characteristics were investigated.

Results: Intention to ST was only predicted by the medical expertise of the tested person, while HPH and HPD were also associated with the application purpose of the test and the presence of an emotionally supporting person. Perceived severity and outcome-expectancy significantly predicted intention to self-test. Technological enthusiastic people had a higher intention to use a self-test.

Conclusions: Intention to ST, HPH and HPD were predicted by different situational and application-related characteristics. Social-cognitive health behaviour theories can be applied to predict self-testing and do not need to be extended by technological affinity.
\end{abstract}

Keywords: Self-testing, Self-diagnosis, Self-management, Health behaviour (theories), Factorial survey

\section{Background}

A broad range of self-tests (testing for e.g. HIV, anaemia, Chlamydia) has become available to the European public via the Internet $[1,2]$. Self-tests can be defined as tests on body materials (e.g. blood, urine, faeces, saliva) that are initiated by consumers to diagnose a particular disorder or risk factor, and that are conducted without the involvement of a health professional [3]. Consumer autonomy, self-management, empowerment, privacy protection and convenience due to the absence of a doctor are mentioned as some of the advantages of self-

\footnotetext{
* Correspondence: gesundheit.praevention@uni-greifswald.de Department Health \& Prevention, Ernst-Moritz-Arndt-University Greifswald, Robert-Blum-Str.13, D-17487 Greifswald, Germany
}

testing (e.g. [2, 4]). On the other hand, disadvantages include concerns about the safety of self-testing, the very low sensitivities displayed by some self-tests [5], the risk of false reassurance in the case of false-negative test results, and the risk of anxiety in the case of true-or false-positive test results as well as unnecessary medical investigations in the case of false-positive results [6-8]. Furthermore, the instruction leaflets of self-tests have been found to be limited on information regarding reliability, follow-up behaviour, and the target group of the test [9].

Despite the potential risks of self-testing, results of surveys from the Netherlands showed that $16 \%$ respondents of a Dutch Internet survey confirmed the use of at 
least one self-test, while in the UK 13\% of the British participants of a written survey had used a self-test at least once $[2,10]$. Similarly, results of a representative survey in Germany of more than 2500 participants showed that $8 \%$ of the German population had used at least one self-test, and about one third of these had used different self-tests [11]. Given the current shortage of physicians in Germany [12], especially in rural areas [13], the need for and use of self-tests could increase in the future. This assumption is furthermore supported by the results of interviews with experts in the development of innovative medical diagnostic devices, who expect a further technological breakthrough of diagnostic devices for end-users in the coming 10 years [14].

The usage of self-tests is embedded in the topics of screening behaviour and disease prevention behaviour in health psychology. Beyond that, however, self-testing presents a new field of application for the validation of common health belief models, which traditionally investigated behaviours such as smoking, alcohol consumption or eating. Although some studies have investigated the psychological determinants of self-testing (e.g. [3, 8, $15,16])$, none of these has considered the technological component of self-testing, for example by integrating technological affinity as an additional predictor for the decision to use a self-test. Thus, this study investigated the role of technological affinity - defined as the attraction to technological devices [17] - when included as an additional predictor into the core concepts of health behaviour theories. It was proposed that the greater the enthusiasm, positive attitude and competence towards technological devices, the greater the intention to use a self-test.

Furthermore, three factors were identified, which in accordance with Hahn and von Lengerke [18] represent the core concepts of health behaviour theories: (a) risk perception, (b) self-efficacy, and (c) outcome expectancy (see Additional file 1: Table S1). Risk perception is a central variable in e.g. the Health Belief Model (HBM), the Protection Motivation Theory (PMT), and the Theory of Planned Behavior (TPB) [19-22]. In the Health Action Process Approach (HAPA), risk perception is composed of (a) the individual's belief of the seriousness of a certain disease/condition (perceived severity), and (b) the individual's belief of the chance of contracting a certain disease/condition (perceived susceptibility) [23]. Additionally, a positive correlation between technological affinity, which is understood as a person's attraction to technological devices [17], and the intention to use a self-test was proposed.

Moreover, the available research has predominantly investigated the psychological determinants of self-testing by conducting interviews or surveys. To the best of our knowledge, no research has been conducted so far to experimentally investigate the impact of situational and application-related characteristics of a test situation on the intention to use a test, such as the test result being displayed immediately on the device, versus the sample being analysed in a laboratory and the result communicated in written form.

Thus, this study had three objectives. First, to experimentally investigate the impact of situational and application-related characteristics of the test situation on the intention to use a self-test (ST) versus being tested by a health professional at home (HPH), or in a doctor's office/hospital (HPD). The HPH setting represents an intermediate scenario between the ST and HPD settings. Second, to investigate whether the core concepts of health behaviour theories can predict the intention to use a self-test. And third, to examine whether taking technological affinity into account as an additional predictor improves the predictive value of the core concepts of health behaviour theories.

\section{Methods \\ Methodological implementation - Factorial survey}

To experimentally investigate the impact of situational and application-related factors, a factorial survey, also called a vignette analysis, was conducted [24]. Vignettes are fictive descriptions of a situation or person, constructed by systematically combining all values of factors (predictors) which are believed to influence a judgment being studied by a rating task (criterion, [24-26]). Vignette analyses are a common method in sociology. In a review of 106 articles from 1982 to 2006, the factorial surveys were most frequently used to measure normative judgments $(n=62)$ and positive beliefs $(n=26)$, they were also often used to examine own (intended) actions $(n=22)$ [26]. The latter is the aim of this survey.

The method of a vignette analysis has a number of advantages for the study presented here. First, vignette analysis is particularly appropriate for investigating context- and condition-related research questions. This is because the respondents, rather than being confronted with abstract values, are presented with concrete and detailed descriptions of a situation, where several different characteristics are systematically varied $[24,26]$. This is especially relevant for the current research question regarding the impact of situational and application-related characteristics of self-testing. Second, in a factorial survey, the principles of an experiment are combined with a social survey $[24,26]$. While the first is associated with a high internal validity, the latter is distinguished by a high external validity. Third, a factorial survey is less subject to social desirability bias than conventional surveys are, because the respondents are not likely to be fully aware of all systematically varied characteristics of a situation, and/or they can be forced to judge two socially equally 
undesired statements at the same time [24, 27]. Finally, factorial surveys are particularly appropriate when researchers want to study actual determinants and combinations of determinants of human judgments, because persons might not be aware of the influences of certain factors on their judgments, and therefore they might not be capable of explicating such influences [28].

\section{Measures - Situational and application-related characteristics}

The final set of situational and application-related characteristics of a test situation was identified using four approaches. First, multiple case histories were developed to cover a broad range of varying kinds of diagnostic test situations within the framework of the research consortium DIA-LOC (http://m-health.psychologie.uni-greifswald.de/dialoc/index.html) [29]. This way, the importance of the factors application purpose and seriousness of the situation was identified, and their values were specified. Second, the literature was reviewed in terms of the application-related characteristics of innovative in-vitro diagnostic devices which can be used outside a laboratory, the so-called lab-on-a-chip systems (LOCs). LOCs are designed for a broad spectrum of application purposes, such as risk assessment, presymptomatic diagnostics, early detection of a disease, and therapy control [30]. Third, an ontology for LOCs was developed within the framework of our research consortium, to further distinguish between the factors application purpose and setting of the test [31]. And finally, the relevance of the previously identified factors was evaluated in a survey by (a) experts in LOC research and development, (b) experts in health technology assessment, and (c) our interdisciplinary research group. Analysis and feedback of the test results, medical expertise of the tested person, and emotional support were identified as three additional factors.

Table 1 gives an overview of the six factors mentioned above with their several values and frequency of occurrence in the factorial survey. Since the factor setting of the test was used as a grouping variable, 337 rated vignettes were related to a ST, 478 to a $\mathrm{HPH}$, and 433 to a HPD situation. For example, the application purpose monitoring of a disease/condition occurred in $16.6 \%$ of the vignettes of the $\mathrm{ST}$, in $15.3 \%$ of the $\mathrm{HPH}$, and in $18.2 \%$ of the HPD group.

Since the given vignette universe of the six situational and application-related factors (Cartesian product: $6 \times 4 \times 3 \times 7 \times 3 \times 3=4536$ ) was far too large to judge all possible combinations, a sample of vignettes (decks of vignettes/subsets) was drawn, and the respondents were presented different selections of the reduced vignette universe. This is a common method in factorial surveys [26, 32]. The reduced vignette universe was selected by a conditional random sampling. To achieve a balanced ratio of the six values of the factor application purpose, 30 vignettes of each value were drawn randomly without replacement, so that the vignette population was reduced to 180 vignettes. Implausible combinations of factor values were deleted before the selection of the vignettes. Figure 1 shows a single vignette of the situation risk assessment. To avoid fatigue and a high number of dropouts, six vignettes were presented to each participant - one vignette of every value of the factor application purpose.

\section{Measures - Psychological characteristics}

Before presenting the vignettes, the sociodemographic characteristics and psychological predictors self-efficacy, perceived susceptibility, and technological affinity were assessed once before the vignettes were presented. The other psychological variables perceived severity and outcome expectancy were presented after each of the six vignettes, because the participants needed to imagine themselves into the vignette scenario to be able to assess these two predictors. The Additional file 1: Table S1 provides an overview of the psychological predictors, their conceptual definitions, items, and answering options. Self-efficacy, which is defined as the individual's confidence in one's capability to successfully perform a certain action, was measured with the well-established General Self-Efficacy scale (GSE, 10 items, $M=28.39, S D=4.11$, Cronbach's $\alpha=.85$, [33]). According to Karrer, Glaser and Clemens [17], technological affinity is defined as a personality trait which is manifested in a positive attitude, enthusiasm, and trust in electronic devices (e.g. mobile phones, computers, personal digital assistants). It was measured by applying three scales, which are all included in the German Technological Affinity Assessment (TA-EG, [17]). Subscale scores were computed and the means calculated for the subscales enthusiasm (5 items, $M=14.46, S D=3.32$, Cronbach's $\alpha=.83$ ), positive attitude (5 items, $M=17.62$, $S D=2.80$, Cronbach's $\alpha=.69)$, and competence towards electronic devices ( 4 items, $M=14.40, S D=2.87$, Cronbach's $\alpha=.74)$. To assess perceived susceptibility, the individual's belief of the chance of contracting a certain disease/condition, the following item was adopted from the 'Berlin Risk Appraisal and Health Motivation Study' (BRAHMS, [34]): the question 'How high do you rate the probability that at some time you will get ..., and its response format 'very unlikely' to 'very likely'. While the BRAHMS project investigated the perceived susceptibility for specific diseases (e.g. risk of heart attack), in this survey, the items were adopted to fit to the vignette factor 'seriousness of a 
Table 1 Overview of situational and application-related vignette dimensions

\begin{tabular}{|c|c|c|c|c|c|c|}
\hline & \multicolumn{2}{|l|}{ ST } & \multicolumn{2}{|l|}{$\mathrm{HPH}$} & \multicolumn{2}{|l|}{$\mathrm{HPD}$} \\
\hline & $\mathrm{n}$ & $\%$ & $\mathrm{n}$ & $\%$ & $\mathrm{n}$ & $\%$ \\
\hline \multicolumn{7}{|l|}{ Application purpose } \\
\hline 1. Risk assessment & 43 & 12.8 & 98 & 20.5 & 67 & 15,5 \\
\hline 2. Early detection of a disease & 57 & 16.9 & 65 & 13.6 & 86 & 19.9 \\
\hline 3. Clinical diagnostics & 66 & 19.6 & 100 & 20.9 & 42 & 9.7 \\
\hline 4. Therapy diagnostics & 34 & 10.1 & 66 & 13.8 & 108 & 24.9 \\
\hline 5. Drug effect & 81 & 24.0 & 76 & 15.9 & 51 & 11.8 \\
\hline 6. Monitoring & 56 & 16.6 & 73 & 15.3 & 79 & 18.2 \\
\hline \multicolumn{7}{|l|}{ Seriousness of the situation } \\
\hline 1. Acute and life-threatening & 115 & 34.1 & 82 & 17.2 & 113 & 26.1 \\
\hline 2. Acute, but not life-threatening & 70 & 20.8 & 138 & 28.9 & 98 & 22.6 \\
\hline 3. Chronic, slowly advancing and life-threatening & 59 & 17.5 & 91 & 19.0 & 89 & 20.6 \\
\hline 4. Chronic, but not life-threatening & 93 & 27.6 & 167 & 34.9 & 133 & 30.7 \\
\hline \multicolumn{7}{|l|}{ Setting of the test } \\
\hline 1. Independently at home without the presence of a health professional & 337 & 27.0 & 0 & 0 & 0 & 0 \\
\hline 2. Tested by a health professional at home & 0 & 0 & 478 & 38.3 & 0 & 0 \\
\hline 3. Tested by a health professional in the doctor's office/hospital & 0 & 0 & 0 & 0 & 433 & 34.7 \\
\hline \multicolumn{7}{|l|}{ Analysis and feedback } \\
\hline 1. Analysed automatically, and the result is displayed immediately & 56 & 16.6 & 105 & 22.0 & 94 & 21.7 \\
\hline $\begin{array}{l}\text { 2. Transmitted automatically and only a conspicuous result is communicated by a } \\
\text { health professional }\end{array}$ & 75 & 22.3 & 0 & 0 & 0 & 0 \\
\hline 3. Transmitted automatically and the result is communicated by a health professional & 125 & 37.1 & 0 & 0 & 0 & 0 \\
\hline 4. Transmitted automatically and the result is communicated in written form & 81 & 24.0 & 0 & 0 & 0 & 0 \\
\hline $\begin{array}{l}\text { 5. Analysed in a laboratory and only a conspicuous result is communicated by a } \\
\text { health professional }\end{array}$ & 0 & 0 & 167 & 34.9 & 130 & 30.0 \\
\hline 6. Analysed in a laboratory and the result is communicated by a health professional & 0 & 0 & 105 & 22.0 & 82 & 18.9 \\
\hline 7. Analysed in a laboratory and the result is communicated in written form & 0 & 0 & 101 & 21.1 & 127 & 29.3 \\
\hline \multicolumn{7}{|l|}{ Medical expertise of the tested person } \\
\hline 1. No & 112 & 33.2 & 126 & 26.4 & 148 & 34.2 \\
\hline 2. Unprofessional & 135 & 40.1 & 167 & 34.9 & 130 & 30.0 \\
\hline 3. Professional & 90 & 26.7 & 185 & 38.7 & 155 & 35.8 \\
\hline \multicolumn{7}{|l|}{ Emotional support } \\
\hline 1. Not present & 120 & 35.6 & 145 & 30.3 & 168 & 38.8 \\
\hline 2. Potentially available & 119 & 35.3 & 149 & 31.2 & 131 & 30.3 \\
\hline 3. Personally present & 98 & 29.1 & 184 & 38.5 & 134 & 30.9 \\
\hline
\end{tabular}

situation'. Thus, the perceived susceptibility of contracting a non-specific 'acute vs. chronic, non-lifethreatening vs. life-threatening' disease was investigated. The four adjusted perceived susceptibility items were summed up to yield a final composite score (4 items, $M=11.96, S D=3.73$, Cronbach's $\alpha=.84$ ).

Perceived severity, the individual's belief of the seriousness of a certain disease/condition, ( 1 item, $M=56.88$, $S D=30.03)$, and outcome expectancy, the individual's weighting of the positive and negative consequences of acting and not acting, ( 1 item, $M=59.53, S D=23.18$ ), were also adopted from BRAHMS. They were adjusted to fit into the fictive vignette scenarios, for example by adding the term '... if the test depicted in the above situation were not conducted', to ensure that the participants imagine themselves in the presented situation before assessing the above two predictors. Furthermore, the criterion intention to use the test was measured after every vignette with the question 'Would you make use of a test that is conducted as described in the situation above?' with a response scale from $1=$ 'certainly not', to $100=$ 'most certainly' (1-item, $M=63.32, S D=29.37$ ). 
Situation: Risk assessment

The diagnostic device could determine immediately if there is a higher risk for a specific chronic, slowly advancing and life-threatening disease.

The test is conducted independently at home without the presence of a health professional. The data are analyzed automatically, and the result is displayed immediately.

The person tested does possess no knowledge to evaluate the test results. A closely related person who could provide emotional support is not present.

Fig. 1 Example of a vignette

\section{Statistical analyses}

The descriptive analyses were conducted using IBM SPSS Statistics 22.0 [35]. Because each participant judged more than one vignette, the vignettes were nested within a person, violating a primary assumption of linear regression analysis, the independence of error values [26]. To solve this problem, multilevel regression models were calculated, allowing for modelling the within (vignette characteristics) and between (respondent characteristics) variance. Four models of increasing complexity were applied with the mixed modelling tool (xtmixed) of the STATA software, using the maximum likelihood estimates (mle option) [36, 37]. The first model was a constant-only empty model without any additional predictors (RIO model). The second model examined the impact of the situational and applicationrelated predictors which were operationalised in the vignettes (RI_V $\mathrm{V}_{\text {all }}$ model). The third model investigated the impact of additional technological affinity (RI_V $\left.\mathrm{V}_{\text {all_}} \mathrm{P}_{\mathrm{TA}}\right)$, and in the fourth model, the health psychological factors were added as predictors (RI_V $\mathrm{V}_{\text {all_}} \mathrm{P}_{\text {all }}$ model). Categorial variables were dummy-coded. Metric variables were centred on the grand mean, prior to entering them into the models.

For each model, the deviances, which indicate how well the models fit the data and which are defined as -2 times the log-likelihood, were calculated ([38], p. 47). Subsequently, using a log rank test, the more complex models were compared to the simpler models regarding their model fit. As a statistic analogous to the multiple $R^{2}$ from ordinary multiple regression analyses, the reduction of the residual error variances in a sequence of models was examined ([38], p. 69-71). In particular, the reduction of the error variance within was calculated in two consecutive models to examine the impact of the situational and application-related factors, and the reduction of the error variance between was calculated in two consecutive models to investigate the impact of technological affinity and the health psychological predictors. Each model was separately calculated for the three settings ST, HPH and HPD, by using the vignette factor setting of the test as a grouping variable.
To facilitate the interpretation of the results, the values of the vignette factors were recoded in such a way that their total mean in dependence of the criterion was in ascending order (see Additional file 2: Table S2). The confounding structure of the parameter estimates was investigated by the alias() function in the statistical programming language $\mathrm{R}$ [39]. This test showed that none of the estimated parameters in our model was confounded with any interaction effect.

\section{Results}

\section{Respondent characteristics}

A random sample of university students were approached via an email distribution list and an online survey was conducted in Germany. Initially, 566 participants started the survey, but there was a remarkable dropout before the vignettes were presented, which implies that a huge proportion of participants decided to decline the survey after they were more familiar with the subject of the study. From those 319 participants who already responded to the first vignette 239 participants completed all six vignettes. Thus, nearly $75 \%$ of all participants, who actively decided to answer the vignettes, had finished this section. Finally, we excluded 31 cases due to potential response bias. In the end, 1248 vignettes, which were rated by 208 students, were included in the analyses. The majority of respondents were female $(76.4 \%)$. The age of the participants varied between 18 and 52 years $(M=23.87, S D=3.86)$. Most of the students $(62.5 \%)$ did not indicate their faculty, but those who did belonged to the following faculties: mathematics and natural sciences (16.8\%), law and economics $(8.7 \%)$, philosophy $(7.2 \%)$, medicine $(3.4 \%)$, and theology $(0.5 \%)$.

\section{Impact of the situational and application-related characteristics}

As a first step, random intercept only models (RIO) with no explanatory variables were calculated for each group (see Additional file 3: Table S3). The RIO model, which estimates the average intention to use a self-test across all vignettes and respondents, was the lowest for the ST group $\left(b_{\mathrm{ST}}=51.51\right)$, higher for the $\mathrm{HPH}$ group 
$\left(b_{\mathrm{HPH}}=65.82\right)$, and the highest for the HPD condition $\left(b_{\mathrm{HPD}}=70.23\right)$. The error variance between amounted to $\delta_{\mathrm{ST}}=218.56, \delta_{\mathrm{HPH}}=302.96$, and $\delta_{\mathrm{HPD}}=217.04$ in the $\mathrm{ST}, \mathrm{HPH}$, and HPD groups, respectively. The error variance within amounted to $\varepsilon_{\mathrm{ST}}=747.95, \varepsilon_{\mathrm{HPH}}=515.65$, and $\varepsilon_{\mathrm{HPD}}=463.74$, respectively. Hence, about $77.4 \%$, $63.0 \%$, and $68.1 \%$ of the total variance of the respective $\mathrm{ST}, \mathrm{HPH}$, and HPD groups was within-person variance, leaving ample room for including predictors.

The second step was to investigate the predictive value of the situational and application-related characteristics on the intention to test, by calculating random intercept models with all vignette factors $\left(\mathrm{RI}_{-} \mathrm{V}_{\text {all }}\right)$. This resulted in a better model fit for the $\mathrm{HPH}$ and HPD groups compared to the empty models $\left(\chi_{\mathrm{HPH}}^{2}=40.54, \mathrm{p}_{\mathrm{HPH}}<0.01 ; \chi_{\mathrm{HPD}}^{2}=35.23\right.$, $\left.\mathrm{p}_{\mathrm{HPD}}<0.05\right)$, but this was not the case for the ST group ( $\chi_{\mathrm{ST}}^{2}=14.92, p=0.78$, see Table 2$)$. However, for all three groups, the deviances and the error variances within were lower in the model with all vignette factors compared to the empty model. Accordingly, in the ST group, the error variance within declined from 747.95 in the RIO model to 672.58 in the RI_V $V_{\text {all }}$ model. This means that about $10.1 \%$ of the error variance within could be explained by adding the situational and application-related predictors to the empty model. For the HPH $10.6 \%$ and for the HPD group $11.2 \%$ of the error variance within were explained by the vignette factors.

Seriousness of the situation and analysis and feedback of the test results did not affect the intention to self-test for any group. Self-test use was significantly predicted by only one vignette factor: medical expertise of the tested person. The intention to use a self-test was on average 13.33 points higher for participants who imagined to have the professional knowledge to evaluate the test results, compared to no knowledge (on a scale from 1 to 100). A professional knowledge compared to no knowledge also significantly increased the intention to be tested by a health professional at home or at a doctor's office $\left(b_{\mathrm{HPH}}=9.80, \mathrm{~b}_{\mathrm{HPD}}=8.66\right)$, but the impact of the medical expertise of the tested person was the highest for the ST group. While the vignette factor application purpose did not significantly influence the intention to use a self-test, it had a significant effect on the HPH and HPD groups, which stated a higher intention to be tested when the application purpose was drug effect or early detection of a disease compared to risk assessment. Additionally, the intention to test was significantly increased for the application purpose monitoring for the HPD group and therapy diagnostics for the HPH group compared to risk assessment. The presence of emotional support affected only the HPH group, whose intention to test was on average 9.71 higher if a closely related person who could provide emotional support was present, compared to the absence of such a person.

\section{Impact of the psychological characteristics}

In the third step, a model with technological affinity added as a predictor (RI_V $V_{\text {all_}} P_{T A}$ ) was calculated (Table 3 ). The addition of this predictor resulted in a better model fit than the RI_ $\mathrm{V}_{\text {all }}$ model for all three settings $\left(\chi_{\mathrm{ST}}^{2}=13.43\right.$, $\mathrm{p}_{\mathrm{ST}}<0.01 ; \chi_{\mathrm{HPH}}^{2}=9.33, \mathrm{p}_{\mathrm{HPH}}<0.05 ; \chi_{\mathrm{HPD}}^{2}=10.58$, $\left.\mathrm{p}_{\mathrm{HPD}}<0.05\right)$. Additionally, the error variance between declined in the ST group from 268.22 to 216.28 ; thus, $19.4 \%$ of this variance was explained by adding technological affinity to the RI_ $\mathrm{V}_{\text {all }}$ model (compared to $8.3 \%$ error variance between of the HPH group and $6.1 \%$ of the HPD group). The results of the RI_V $\mathrm{V}_{\text {all_}} \mathrm{P}_{\mathrm{TA}}$ model showed that, while the intention to use a self-test significantly increased with higher values on the technological affinity enthusiasm scale $\left(\mathrm{b}_{\mathrm{ST}}=6.21\right)$, the intention of being tested by a health professional at a doctor's office/hospital significantly increased with higher values on the technological affinity positive attitude scale $\left(b_{\text {HPD }}=7.29\right)$. However, the impact of the situational and application-related characteristics on the intention to test did not change when adding the technological affinity scales to the model.

Finally, in the fourth model, the health psychological factors were added as predictors (RI_V $\mathrm{V}_{\text {all_}} \mathrm{P}_{\text {all }}$, see Table 3 ). This addition resulted in a better model fit than the RI_Vall $\mathrm{P}_{\mathrm{TA}}$ model for all three settings $\left(\mathrm{X}_{\mathrm{ST}}^{2}=274.94\right.$, $\mathrm{p}_{\mathrm{ST}}<0.001 ; \chi_{\mathrm{HPH}}^{2}=259.33, \mathrm{p}_{\mathrm{HPH}}<0.001 ; \chi_{\mathrm{HPD}}^{2}=262.05$, $\left.\mathrm{p}_{\mathrm{HPD}}<0.001\right)$. The error variance within declined from the RI_V $\mathrm{V}_{\text {all_ }} \mathrm{P}_{\mathrm{TA}}$ to the RI_ $\mathrm{V}_{\text {all_}} \mathrm{P}_{\text {all }}$ model in all three test settings. About $47.7 \%, 41.57 \%$ and $52.35 \%$ of the error variance within in the ST, HPH and HPD groups, respectively, could be explained by adding the health psychological predictors to the RI_ $\mathrm{V}_{\text {all_}} \mathrm{P}_{\mathrm{TA}}$ model.

Perceived severity $\left(\mathrm{b}_{\mathrm{ST}}=0.16, \mathrm{~b}_{\mathrm{HPH}}=0.23, \mathrm{~b}_{\mathrm{HPD}}=0.19\right)$ and outcome expectancy $\left(\mathrm{b}_{\mathrm{ST}}=0.89, \mathrm{~b}_{\mathrm{HPH}}=0.68\right.$, $\left.\mathrm{b}_{\mathrm{HPD}}=0.69\right)$ significantly predicted the intention to test for all three test settings. Furthermore, in the RI_ $\mathrm{V}_{\text {all_}} \mathrm{P}_{\text {all }}$ model, the impact of technological affinity and the vignette factor application purpose disappeared, whereas the vignette factor analysis and feedback of the test results had a significant impact on the HPH and HPD groups. However, the professional expertise of the tested person remained a significant predictor of the intention to self-test $\left(\mathrm{b}_{\mathrm{ST}}=7.17\right)$ and being tested by a health professional at home $\left(\mathrm{b}_{\mathrm{HPH}}=7.04\right)$.

\section{Discussion}

\section{Main findings and comparison with other studies}

This study had three objectives. First, the impact of situational and application-related characteristics of the test 
Table 2 Multilevel model with vignette characteristics and the criterion "intention to use a test" separately for the groups ST, HPH, and HPD

\begin{tabular}{|c|c|c|c|c|c|c|}
\hline \multirow[b]{3}{*}{ Fixed effects } & \multicolumn{6}{|c|}{ RI_Vall } \\
\hline & \multicolumn{2}{|l|}{ ST } & \multicolumn{2}{|l|}{$\mathrm{HPH}$} & \multicolumn{2}{|l|}{ HPD } \\
\hline & $b$ & (SE) & $b$ & (SE) & $b$ & (SE) \\
\hline intercept & $36.00^{* * *}$ & $(7.03)$ & $43.68^{* * *}$ & (5.08) & $55.24^{* * *}$ & $(4.48)$ \\
\hline \multicolumn{7}{|l|}{ Vignette characteristics } \\
\hline \multicolumn{7}{|l|}{ Application purpose } \\
\hline \multicolumn{7}{|l|}{ Risk assessment ${ }^{\text {RefA }}$} \\
\hline Clinical diagnostics & 0.68 & $(6.31)$ & 6.29 & (3.54) & 5.86 & $(5.08)$ \\
\hline Drug effect & 1.08 & $(6.50)$ & $8.53^{*}$ & $(3.67)$ & $9.58^{*}$ & $(4.64)$ \\
\hline Early detection of a disease & -1.57 & $(6.65)$ & $8.62^{*}$ & $(4.27)$ & $13.22^{* * *}$ & (3.86) \\
\hline Monitoring & 6.40 & $(6.47)$ & 7.93 & (4.19) & $11.78^{* *}$ & $(4.20)$ \\
\hline Therapy diagnostics & 6.50 & $(7.74)$ & $8.98^{*}$ & (3.89) & 5.86 & $(5.08)$ \\
\hline \multicolumn{7}{|l|}{ Seriousness of the situation } \\
\hline \multicolumn{7}{|l|}{ Acute and life-threatening RefB } \\
\hline Acute, but not life-threatening & 6.66 & $(5.37)$ & 2.96 & (3.97) & -4.08 & $(4.11)$ \\
\hline Chronic, but not life-threatening & 6.67 & $(5.60)$ & 3.44 & (3.87) & -0.096 & (3.59) \\
\hline Chronic, slowly advancing and life-threatening & 2.30 & $(5.51)$ & 6.19 & (4.30) & 2.00 & (3.99) \\
\hline \multicolumn{7}{|l|}{ Analysis and feedback } \\
\hline \multicolumn{7}{|l|}{ Transmitted automatically and the result is communicated in written form RefC } \\
\hline $\begin{array}{l}\text { Transmitted automatically and only a conspicuous result is communicated } \\
\text { by a health professional }\end{array}$ & RefC -1.57 & $(5.77)$ & & & & \\
\hline Transmitted automatically and the result is communicated by a health professional & $\operatorname{RefC}_{1.40}$ & $(5.45)$ & & & & \\
\hline \multicolumn{7}{|l|}{$\begin{array}{l}\text { Analyzed in a laboratory and only a conspicuous result is communicated by } \\
\text { a health professionaleff }\end{array}$} \\
\hline Analyzed automatically, and the result is displayed immediately & $\operatorname{RefC}_{-5.41}$ & $(5.27)$ & $\operatorname{RefD}_{4.58}$ & (3.28) & $\operatorname{RefD}_{3.46}$ & (3.44) \\
\hline Analyzed in a laboratory and the result is communicated in written form & & & $\operatorname{RefD}_{4.31}$ & (3.34) & RefD -1.75 & (3.25) \\
\hline Analyzed in a laboratory and the result is communicated by a health professional & & & ${ }^{R e f D} 6.21$ & (3.53) & $\operatorname{RefD}_{5} .68$ & (3.55) \\
\hline \multicolumn{7}{|l|}{ Medical expertise of the tested person } \\
\hline \multicolumn{7}{|l|}{ No RefE } \\
\hline Unprofessional & 4.42 & $(4.94)$ & 0.96 & (3.16) & 1.09 & (3.10) \\
\hline Professional & $13.33^{* *}$ & $(4.96)$ & $9.80^{* *}$ & (3.12) & $8.66^{* *}$ & (3.02) \\
\hline \multicolumn{7}{|l|}{ Emotional support } \\
\hline \multicolumn{7}{|l|}{ Not present ${ }^{\text {RefF }}$} \\
\hline Potentially available & 7.93 & $(4.60)$ & 5.25 & $(3.27)$ & 3.52 & (3.18) \\
\hline Personally present & 8.64 & $(4.87)$ & $9.71^{* *}$ & (3.14) & 1.76 & (3.66) \\
\hline \multicolumn{7}{|l|}{ Random effects } \\
\hline$\delta_{\text {im }}$ (error variance between) & 268.22 & & 303.78 & & 227.83 & \\
\hline$\varepsilon_{\mathrm{ij}}$ (error variance within) & 672.58 & & 461.16 & & 411.73 & \\
\hline Deviance & 3247.86 & & 4468.87 & & 3985.02 & \\
\hline$N_{O} / N_{G}$ & $337 / 183$ & & $478 / 196$ & & $433 / 192$ & \\
\hline
\end{tabular}

$\mathrm{N}_{\mathrm{o}}=$ Number of observations/vignettes

$\mathrm{N}_{\mathrm{G}}=$ Number of groups/respondents

${ }^{*} p<0.05,{ }^{* *} p<0.01,{ }^{* * *} p<0.001$

situation on the intention to use a self-test (ST) versus being tested by a health professional at home (HPH), or in a doctor's office/hospital (HPD), were experimentally investigated. A factorial survey was conducted enabling the systematic variation of a set of situational and application-related characteristics. The results suggested 


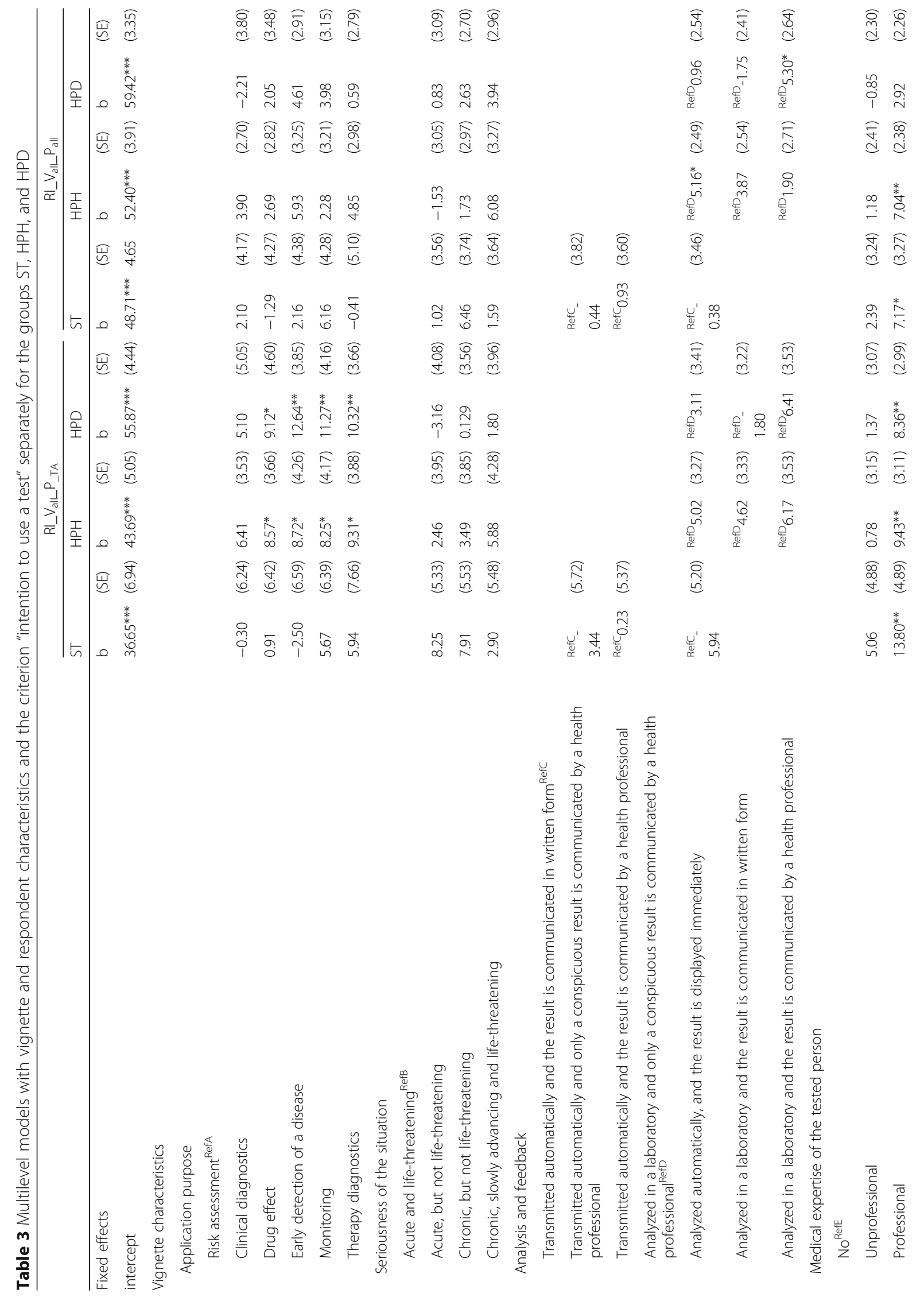




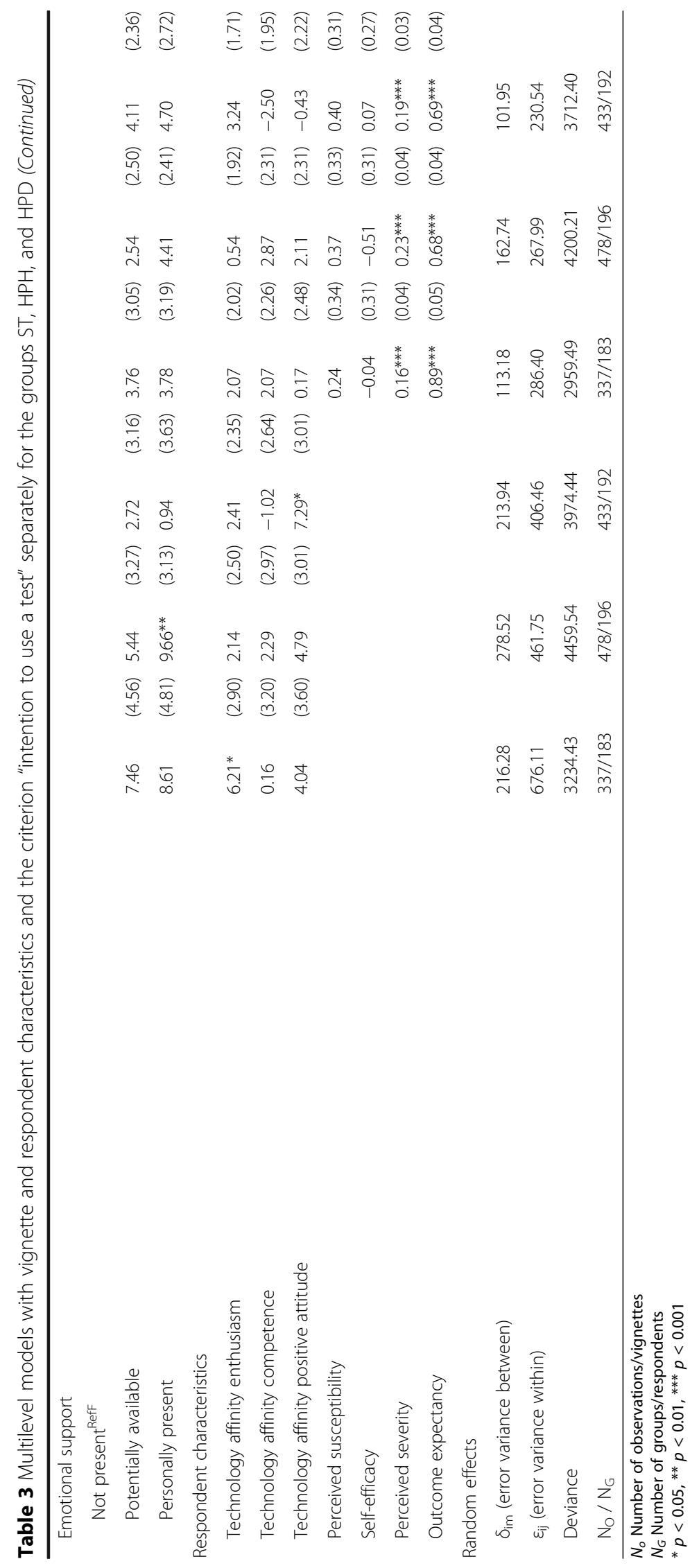


that the intention to use a self-test was only predicted by the medical expertise of the tested person. Exclusively participants who were asked to imagine themselves as having the professional knowledge to evaluate the test results had a higher intention to use a self-test than those who had no medical knowledge. Professional medical expertise was also important, though to a lesser degree, in the decision of being tested by a health professional at home or at a doctor's office. Presumably, participants did not think that it made sense to conduct a test by themselves or by a health professional if they do not understand the test results. While the seriousness of the situation and the analysis and feedback of the test results did not predict any intention to test, the application purpose did influence the decision whether to be tested by a health professional at home or at a doctor's office. Interestingly, the presence or absence of a closely related person who could provide emotional support did not affect the intention to self-test, but the presence of a supportive person did raise the probability of the intention of being tested by a health professional at home.

The second objective of this study was to investigate whether the core concepts of health behaviour theories can predict the intention to use a self-test. The results showed that there was no significant association between perceived susceptibility and the intention to self-test, to be tested at home, or at a doctor's office. However, previous research on the psychological determinants of selftesting for cholesterol, glucose and HIV in a crosssectional survey has found that perceived susceptibility was a significant predictor of the use of cholesterol and HIV self-tests, but not of glucose [3]. The relationship between perceived susceptibility and the intention to use a self-test therefore seems to depend on the specific health risk or disease under investigation. Since our factorial survey did not specify the health risk or disease, but instead focussed on the participants' views on the chance of contracting a non-specific 'acute vs. chronic, non-life-threatening vs. life-threatening' disease, the association between perceived susceptibility and the intention to test did not become apparent.

Although in previous research, self-efficacy has been shown to be an important predictor of the intention to attend and actual attendance of screening programmes, as well as of self-testing for cholesterol, glucose and HIV [3, $20,40,41]$, in our factorial survey no such associations were found. However, in our survey self-efficacy was assessed with the GSE, whereas in previous research the items to assess self-efficacy were specifically related to self-testing [3]. A question is whether standardized instruments should be adopted for the specific health behaviour.

In comparison, results for perceived severity and outcome expectancy were in accordance with the theoretical predictions, because both significantly predicted the intention to be self-tested and tested by a health professional at home or at a doctor's office. In contrast, Grispen et al. [3] found no association between perceived severity and self-testing for glucose, HIV or cholesterol. According to Hahn and Lengerke [18], outcome expectancy is equivalent to perceived barriers and perceived benefits from the HBM or the response efficacy from the PMT. While perceived benefits significantly predicted the use of all three self-tests, test-specific associations were identified for response efficacy and perceived barriers [3].

The third objective was to investigate whether the predictive value of the core concepts of health behaviour theories can be improved by adding technological affinity. The results showed that the addition of technological affinity to the situational and application-related factors significantly predicted the intention to self-test, which supported our assumption about the positive relationship between technological affinity and self-testing to some degree. Additionally, the hypothesised benefit of assessing technological affinity with different subscales was confirmed, because the intention to use a self-test was only predicted by a higher technical enthusiasm, whereas people who assigned themselves no technological competence, but still had a positive attitude towards technology, preferred to be tested at a doctor's office. However, when adding the health psychological predictors to the final model, technological affinity turned out to be statistically non-significant. This suggests that the health psychological predictors incorporated and superseded the predictive value of technological affinity.

\section{Strengths and limitations}

Self-efficacy was measured with the GSE scale, which enabled the comparison of the results with those of other studies. However, a phrasing in terms of the individual's confidence in one's capability to successfully perform a self-test would have been more sensitive and in line with the theoretical assumptions. Second, as perceived susceptibility was adopted to fit to the vignette factor 'seriousness of a situation', this study has investigated the perceived susceptibility of getting a non-specific 'acute vs. chronic, non-life-threatening vs. life-threatening' disease. In future studies, however, the individual's belief of the chance of contracting $a$ certain disease/condition should also be investigated, since significant associations might depend on the specification of the disease/condition to be tested. Third, a factorial survey was chosen because it allows an experimental investigation of the impact of situational and application-related factors. However, the display of fictive scenarios might be an additional 
reason for why there was no or only little association between self-efficacy and perceived susceptibility with the intention to test. The results may also have had a low external validity, but they are distinguished by a high internal validity. Fifth, order effects cannot be excluded. This study aimed at a balanced ratio of the six values of the factor application purpose, so that 30 vignettes of each of its values were drawn randomly without replacement. Sixth, the sample consisted only of university students, who, compared to the general population, may have specific characteristics such as a higher education level or a higher family income. On the other hand, a homogenous sample is advantageous for experimental investigations, because they are less biased. Consequently, future work should re-examine the research questions posed here by comparing actual self-testers with non-self-testers in the general population.

\section{Conclusions}

Despite the abovementioned limitations of this study, it can be concluded that the situational and applicationrelated determinants which predicted the intention to use a self-test differed from those predicting the intention of being tested by a health professional at home or in a doctor's office/hospital. In fact, the only situational and application-related factor which predicted the intention to self-test was a professional medical expertise of the tested person. Although the most frequently stated advantages of self-testing include the faster diagnostics and higher privacy protection [14], situational and application-related factors such as 'analysed automatically, and the result is displayed immediately' did not significantly predict the intention to use a self-test. Additionally, technological affinity predicted the intention to self-test, but when the core concepts of social-cognitive health behaviour theories were added, the impact of technological affinity was incorporated. Therefore, it can be concluded that the existing socialcognitive health behaviour theories can be applied to predict the intention to use a self-test and do not need to be extended by technological affinity. However, since vignettes were used to investigate the determinants of the intention to use a self-test, additional studies comparing actual self-testers with non-self-testers are necessary to fully understand the psychological, situational and application-related determinants of self-test use.

\section{Additional files}

Additional file 1: Table S1. Overview of psychological constructs, conceptual definitions, items, and answering options. Description: This file contains the additional Table 1 which gives an overview of the psychological constructs, conceptual definitions, items, and answering options of the survey. (DOC $69 \mathrm{~kb}$ )

Additional file 2: Table S2. Descriptive statistics of the vignette characteristics in dependence of the criterion "intention to test" separately for the groups ST, HPH, and HPD. Description: This file contains the additional Table 2 which gives an overview of the descriptive statistics of the vignette characteristics in dependence of the criterion "intention to test" separately for the groups ST, HPH, and HPD. (DOC $91 \mathrm{~kb}$ )

Additional file 3: Table S3. Random intercept only model for the criterion "intention to use a test" separately for the groups ST, HPH, and HPD". Description: This file contains the additional Table 3 which shows the results of the random intercept only model for the criterion intention to use a test separately for the groups ST, HPH, and HPD. (DOC $69 \mathrm{~kb}$ )

Additional file 4: Data_Online Survey_Determinants of intention to self-test.SAV". Description: The file contains the data of the survey. (SAV $79 \mathrm{~kb})$

\section{Abbreviations}

BRAHMS: Berlin Risk Appraisal and Health Motivation Study; GSE: General Self-Efficacy scale; HAPA: Health Action Process Approach; HBM: Health Belief Model; HPD: Health professional at a doctor's office; HPH: Health professional at home; LOCs: Lab-on-a-chip systems; PMT: Protection Motivation Theory; RI_Vall: Random intercept models with all vignette factors; RI_ $V_{\text {all_}} P_{\text {all: }}$ : Random intercept models with all vignette factors and all psychological factors; RI_Vall_PTA: Random intercept models with all vignette factors and technological affinity; RIO: Random intercept only models; ST: Self-test; TA-EG: German Technological Affinity Assessment; TPB: Theory of Planned Behavior

\section{Acknowledgements}

This work was supported by the German Federal Ministry of Education and Research, Berlin [grant number: 01GP1005A].

\section{Funding}

The source of funding did not influence the study design, the writing of the manuscript, or the decision to submit the manuscript for publication.

Availability of data and materials

Additional files 1,2 and 3 contain the questionnaires and additional analysis. The Additional file 4 contains the data.

\section{Authors' contributions}

PK, TR, HM have made substantial contributions to conception and design of the survey, and acquisition of data. PK and TR conducted the analysis of the data. PK interpreted the results and drafted the manuscript. HM and SiS revised the manuscript critically for important intellectual content. All authors read and approved the final manuscript.

\section{Competing interests}

The authors declare that they have no competing interests.

\section{Consent for publication}

Not applicable.

\section{Ethics approval and consent to participate}

This study is exempt from ethics approval because it was conducted in compliance with all federal regulations governing the protection and privacy of human subjects and in accordance with the ethical principles of the German Psychological Society [42] as well as the ethical codex of the German Sociological Society [43]. Research carried out was in compliance with the Helsinki Declaration [44]. The participants gave consent to participate in the survey and permission to publish the research results.

\section{Publisher's Note}

Springer Nature remains neutral with regard to jurisdictional claims in published maps and institutional affiliations. 
Received: 20 August 2015 Accepted: 16 June 2017

\section{Published online: 10 July 2017}

\section{References}

1. Ryan A, Wilson S, Greenfield S, Clifford S, McManus R, Pattison H. Range of self-tests available to buy in the United Kingdom: an internet survey. J Public Health. 2006;28(4):370-4.

2. Ronda G, Portegijs P, Dinant G-J, Buntinx F, Norg R, van der Weijden T. Use of diagnostic self-tests on body materials among internet users in the Netherlands: prevalence and correlates of use. BMC Public Health. 2009;9(1):100

3. Grispen J, Ronda G, Dinant G-J, de Vries N, van der Weijden T. To test or not to test: a cross-sectional survey of the psychosocial determinants of self-testing for cholesterol, glucose, and HIV. BMC Public Health. 2011;11(1):112.

4. Ickenroth MHP, Ronda G, Grispen JEJ, Dinant G-J, de Vries N, van der Weijden T. How do people respond to self-test results? A cross-sectional survey. BMC Fam Pract. 2010;11:77.

5. Michel C-EC, Saison FG, Joshi H, Mahilum-Tapay LM, Lee HH. Pitfalls of internetaccessible diagnostic tests: inadequate performance of a CE-marked Chlamydia test for home use. Sex Transm Infect. 2009:85(3):187-9.

6. Ryan A, Greenfield S, McManus R, Wilson S. Self-care: has DIY gone too far? Br J Gen Pract. 2006;56(533):907-8.

7. Ryan A, Wilson S, Taylor A, Greenfield S. Factors associated with self-care activities among adults in the United Kingdom: a systematic review. BMC Public Health. 2009;9(1):96.

8. Ickenroth MHP, Grispen J, Ronda G, Tacken M, Dinant G-J, de Vries NK, van der Weijden T. Motivation and experiences of self-testers regarding tests for cardiovascular risk factors. Health Expect. 2014;17(1):60-72.

9. Grispen J, Ickenroth MHP, de Vries NK, van der Weijden T, Ronda G. Quality and use of consumer information provided with home test kits: room for improvement. Health Expect. 2014;17(5):741-52.

10. Ryan A, Wilson S, Greenfield S. Prevalence of the use of self-tests by adults in the United Kingdom: a questionnaire survey. J Public Health. 2010;32(4):519-25.

11. Muehlan H, Kuecuekbalaban P, Schmidt S. Diagnostische Direct-toConsumer-Tests - Einstellungen, Verfügbarkeit, Inanspruchnahme [Diagnostic direct-to-consumer-Tests - Attitudes, availability, utilisation]. In: eHealth 2015 Informations- und Kommunikationstechnologien im Gesundheitswesen. Edited by Duesberg F. Solingen: medical future; 2015: 164-166.

12. Kopetsch $\mathrm{T}$. The medical profession in Germany: past trends, current state and future prospects. Cah Sociol Demogr Med. 2004;44(1):43-70.

13. Natanzon I, Szecsenyi J, Ose D, Joos S. Future potential country doctor: the perspectives of German GPs. Rural Remote Health. 2010;10(2):1347.

14. Kuecuekbalaban P, Schmidt S, Kraft K, Hoffmann W, Muehlan H. Exploring risks and benefits of point-of-care tests for healthcare and self-tests for laypersons: an interview study assessing complementary expert perspectives on diagnostic lab-on-a-chip systems. Technol Health Care. 2014;22(6):817-33.

15. Ryan A, Greenfield S, Wilson S. Prevalence and determinants of the use of self-tests by members of the public: a mixed methods study. BMC Public Health. 2006;6(1):1-5.

16. Ryan A, Ives J, Wilson S, Greenfield S. Why members of the public self-test: an interview study. Fam Pract. 2010;27(5):570-81.

17. Karrer K, Glaser C, Clemens C, Bruder C. Technikaffinität erfassen Der Fragebogen TA-EG [measuring technological affinity - the questionnaire TA-EG]. In: Lichtenstein A, Stößel C, Düsseldorf CC, editors. Der Mensch als Mittelpunkt technischer Systeme. Volume 8. Berliner Werkstatt Mensch-Maschine-Systeme. Germany: VDI Verlag GmbH; 2009. p. 196-201.

18. Hahn A, Von Lengerke T: Evaluating a cholesterol screening: Risk appraisals, outcome expectancies, and self-efficacy beliefs as predictors of physical exercise and alcohol consumption. In: Advances in health psychology research. Volume 1, Edited by Schwarzer R. Berlin: Freie Universität; 1998.

19. Becker MH. The health belief model and personal health behavior. Thorofare, NJ: Slack; 1974.

20. Rosenstock IM. The health belief model: explaining health behavior through expectancies. In: Glanz K, Lewis FM, Rimer BK, editors. Heatlth behavior and health education: theory, research, and practice. San Francisco, CA: JosseyBass; 1990. p. 39-62.
21. Rogers RW. Cognitive and physiological processes in fear appeals and attitude change: a revised theory of protection motivation. In: Cacioppo JR, Petty RE, editors. Social psychophysiology: a sourcebook. New York, NY: Guilford Press; 1983. p. 153-76.

22. Ajzen I. The theory of planned behaviour. Organ Behav Hum Decis Process. 1991;50(2):179-211.

23. Lippke S, Renneberg B. Theorien und Modelle des Gesundheitsverhaltens [Theories and models of health behavior]. In: B. Renneberg \& P. Hammelstein (Eds.), Gesundheitspsychologie. Heidelberg: Springer; 2006:35-60.

24. Steiner $\mathrm{P}$, Atzmüller $\mathrm{C}$. Experimentelle Vignettendesigns in faktoriellen Surveys. KZfSS Kölner Zeitschrift für Soziologie und Sozialpsychologie. 2006;58(1):117-46.

25. Hechter M, Ranger-Moore J, Jasso G, Horne C. Do values matter? An analysis of advance directives for medical treatment. Eur Sociol Rev. 1999:15(4):405-30.

26. Wallander L. 25 years of factorial surveys in sociology: a review. Soc Sci Res. 2009:38(3):505-20

27. Gross C, Kriwy P. Fairness Judgement on the allocation of organ donations. Results of a factorial survey. Gesundheitswesen. 2008;70(8/9):541-9.

28. Alexander CS, Becker HJ. The use of vignettes in survey research. Public Opin Q. 1978:42(1):93-104.

29. Brendel A, Spies C, Dierks C. Rechtlicher Anpassungsbedarf für diagnostische Lab-on-a-chip-Systeme. Medizinrecht. 2015;33(5):321-7.

30. Bier FF, Schumacher S. Biosensoren der Zukunft: Patientennahe in vitroDiagnostik für personalisierte Medizin. Public Health Forum. 2011;19(1):26. e21-26.e24

31. Nussbeck G, Soltani N, Denecke K. Making knowledge on healthcare technologies understandable: an ontology for lab-on-a-chip systems. Stud Health Technol Inform. 2013;192:972.

32. Auspurg K, Hinz T, Liebig S, Sauer C. Wie unplausibel darf es sein? Der Einfluss von Designmerkmalen auf das Antwortverhalten in Faktoriellen Surveys. In: Unsichere Zeiten: Herausforderungen gesellschaftlicher Transformationen. Volume 34. Edited by Soeffner H-G. Wiesbaden: VS-Verlag; 2010

33. Schwarzer R, Jerusalem M. Generalized self-efficacy scale. In: Weinman J, Wright S, Johnston M, editors. Measures in health psychology: a user's portfolio causal and control beliefs. Windsor, UK: NFER-NELSON; 1995. p. 35-7.

34. Renner B, Hahn A, Schwarzer R. Risiko und Gesundheitsverhalten. Dokumentation der Meßinstrumente des Forschungsprojekts "Berlin risk Appraisal and Health Motivation Study" (BRAHMS). [Risk and health behaviour. Documentation of the measuring instruments of the research project "Berlin risk Appraisal and Health Motivation Study" (BRAHMS).]. Berlin: Freie Universität Berlin; 1996.

35. IBM Corp. IBM SPSS Statistics for Windows, Version 22.0. In. Armonk, NY, USA 2013

36. Stata Corp. Stata Statistical Software: Release 11. College Station. TX, USA: StataCorp LP; 2013.

37. Rabe-Hesketh S, Skrondal A. Multilevel and longitudinal modeling using Stata. College Station: Stata Press; 2005.

38. Hox J. Multilevel Analysis: Techniques and Applications, Second Edition. New York, NY: Taylor \& Francis; 2010

39. R Core Team. R: A language and environment for statistical computing. $R$ Foundation for Statistical Computing. Vienna: R Core Team; 2013.

40. Cooke R, French DP. How well do the theory of reasoned action and theory of planned behaviour predict intentions and attendance at screening programmes? A meta-analysis. Psychol Health. 2008;23(7):745-65.

41. Milne SE, Orbell S. Can Protection Motivation Theory predict breast selfexamination? In: Understanding and changing in health behaviour: From health beliefs to self-regulation. Volume 2. Edited by Abraham C, Conner M, Norman P. London, UK: Psychology Press; 2000:51-72.

42. Ethical Principles of the German Psychological Society and the Association of German Professional Psychologists. 2014. http://www.bdp-verband.org/ bdp/verband/ethik.shtml.

43. Code of ethics of the German Sociological Association and the Berufsverbandes Deutscher Soziologen. 2014. http://www.soziologie. de/en/gsa/ethik/code-of-ethics.html.

44. Ethical Principles for Medical Research Involving Human Subjects. 2014. http://www.wma.net/en/30publications/10policies/b3/index.html. 